\title{
ANÁLISIS BIBLIOMÉTRICO DE LAS PUBLICACIONES CIENTÍFICAS ORIGINALES DEL INSTITUTO NACIONAL DE SALUD DEL PERÚ EN EL PERIODO 1998-2018
}

\author{
Franco Romaní (i1),a \\ 1 Oficina General de Investigación y Transferencia Tecnológica, Instituto Nacional de Salud, Lima, Perú. \\ a Médico cirujano
}

\section{RESUMEN}

Objetivos: Medir la producción científica del Instituto Nacional de Salud (INS) mediante indicadores bibliométricos de producción, colaboración e impacto. Materiales y métodos: Estudio bibliométrico de publicaciones originales en revistas indizadas en Scopus y Scielo Perú en el periodo 1998-2018. Los indicadores de producción fueron el número de publicaciones por año y por subperiodos (1998-2008 y 20092018), por temática, y por revista de publicación. Los indicadores de colaboración fueron el índice de coautoría, el índice de número de firmas institucionales, la tasa de colaboración nacional e internacional, la proporción de primeria autoría con filiación del INS. Los indicadores de impacto fueron el número de citaciones por año, índice citas/documento, la proporción de documentos alguna vez citados y la velocidad de citación. Resultados: 618 publicaciones tuvieron al menos un autor con filiación INS. El 55,9\% (346/618) fue publicado en revistas científicas peruanas. 30 autores del INS fueron grandes productores. El 49,0\% (303/618) de las publicaciones tuvo al primer autor con filiación INS. $132(21,4 \%)$ publicaciones fueron sobre enfermedades transmitidas por vectores; $9,7 \%$ (60), sobre tuberculosis; y 9,5\% (59), sobre zoonosis. La tasa de colaboración internacional fue de 38,8\%. La razón citas/documentos fue de 12,8 para todo el periodo. El índice $\mathrm{H}$ de las publicaciones con participación del INS fue 37. Conclusiones: En las últimas dos décadas, la contribución del INS estuvo centrada en las enfermedades infecciosas. Por otro lado, requiere fortalecer los índices de productividad de sus autores, así como consolidar las nuevas líneas temáticas que han desarrollado en la última década. Este esfuerzo permitirá mejorar su función como instituto público de investigación.

Palabras clave: Bibliometría; Autoría y Coautoría en la Publicación Científica; Institutos de Investigación; Investigación; Indicador de Colaboración; Perú (Fuente: DeCs BIREME).

\section{BIBLIOMETRIC ANALYSIS OF ORIGINAL SCIENTIFIC PUBLICATIONS FROM THE INSTITUTO NACIONAL DE SALUD DEL PERU FROM 1998 TO 2018}

\section{RESUMEN}

Objetives: To measure the scientific production of the Instituto Nacional de Salud del Perú(INS) through bibliometric indicators of production, collaboration and impact. Materials and methods: Bibliometric study of original publications from journals indexed in Scopus and Scielo Peru in the period between 1998 and 2018. The production indicators were: the number of publications per year and per subperiod (1998-2008 and 2009-2018), by theme, and by journal. The collaboration indicators were: the co-authorship index, the number of institutional signatures, the national and international collaboration rate, the proportion of first-time authorship with INS affiliation. The impact indicators were: the number of citations per year, citation/document index, the proportion of documents ever cited and the citation speed. Results: A total of 618 publications had at least one author with INS affiliation. From these, 55.9\% (346/618) were published in Peruvian scientific journals and 30 INS authors were major producers. From the publications total, $49.0 \%$ (303/618) had a first author with INS affiliation. Regarding the subject of investigation, 132 (21.4\%) publications were on vector-borne diseases; 9.7\% (60), on tuberculosis; and $9.5 \%$ (59), on zoonoses. The international collaboration rate was $38.8 \%$. The ratio of citations/documents was 12.8 for the entire period. The $\mathrm{H}$ index of publications with INS participation was 37 . Conclusions: In the last two decades, the contribution of the INS was focused on infectious diseases. On the other hand, it is required to strengthen the productivity indexes of INS authors, as well as consolidating the new thematic lines that they have developed in the last decade. This effort will allow the INS to improve its function as a public research institute.

Palabras clave: Bibliometría; Autoría y Coautoría en la Publicación Científica; Institutos de Investigación; Investigación; Indicador de Colaboración; Perú (Source: MeSH NLM). 


\section{INTRODUCCIÓN}

En las últimas décadas, la producción científica de los centros de investigación del Perú ha crecido en todas las áreas del conocimiento ${ }^{(1)}$, incluso el campo biomédico y salud ${ }^{(2,3)}$. Los estudios bibliométricos utilizan métodos estadísticos para cuantificar y describir las características de la literatura científica ${ }^{(4)}$. La bibliometría ha caracterizado sistemáticamente las publicaciones científicas de autores e instituciones peruanas en periodos temporales definidos, por temática ${ }^{(5-7)}$ y con fines de descripción, evaluación o monitoreo ${ }^{(8,9)}$.

El Instituto Nacional de Salud (INS) es un instituto público de investigación perteneciente al Ministerio de Salud del Perú (MINSA). Su misión es desarrollar investigación científica en el campo de la salud pública, así como difundirla para mejorar la calidad de vida de la población. Algunos estudios han cuantificado la producción científica del INS. Entre el 2007 y el 2011, el MINSA tuvo 457 publicaciones, sin embargo, no es posible identificar en cuántas participó el INS ${ }^{(1)}$. Un análisis en la base de datos Web of Science (WoS) para el periodo 1997-2016 encontró 196 publicaciones con participación del INS ${ }^{(2)}$. Otro estudio de la producción científica peruana en medicina en revistas indizadas en WoS, encontró 65 publicaciones con filiación del INS entre el 2000 y el $2009^{(3)}$.

No encontramos estudios específicos sobre la producción científica del INS, por lo que desconocemos las características de sus publicaciones, tendencias en el tiempo e impacto considerando sus citaciones. El análisis de dos décadas de dichos indicadores bibliométricos permitirá cubrir apropiadamente esta brecha en el conocimiento ${ }^{(2)}$. Por lo descrito, realizamos un estudio bibliométrico de las publicaciones científicas originales con participación del INS en el periodo 1998-2018.

\section{MATERIALES Y MÉTODOS}

\section{Diseño y fuente de información}

Se realizó un estudio bibliométrico de la producción científica del INS del Perú mediante el análisis de sus publicaciones originales en revistas indizadas en Scopus y Scielo Perú en el periodo 1998-2018.

Se seleccionó la base de datos Scopus porque incluye todas las revistas indizadas en Medline ${ }^{(4)} \mathrm{y}$ tiene una importante colección de revistas latinoamericanas, además, permite la búsqueda por filiación institucional e identificar la filiación de todos los autores, lo cual es necesario para los indicadores de colaboración ${ }^{(10)}$. Scielo Perú permitió incluir las publicaciones en revistas peruanas no indizadas en Scopus.

La búsqueda fue realizada en octubre del 2019. Para la recuperación en Scopus, se usó la búsqueda por afiliación al «Instituto Nacional de Salud, Lima» (identificador de afiliación: 60071247). Se filtró para el periodo 1998-2018; pos-

\section{MENSAJES CLAVE}

Motivación para realizar el estudio: Se ha cuantificado la producción científica biomédica en el Perú, sin embargo, no se ha evaluado la producción del Instituto Nacional de Salud (INS) del Perú.

Principales hallazgos: El INS participó en 618 publicaciones originales. A lo largo de los años ha incrementado su producción y cada vez tiene más colaboración internacional y citaciones, sin embargo, su liderazgo se ha reducido. Los tres temas más abordados fueron enfermedades transmitidas por vectores, tuberculosis y zoonosis.

Implicancias: El Perú requiere de un instituto de investigación en salud pública fortalecido y con liderazgo a nivel nacional e internacional.

teriormente se descargó la base de datos en formato .CSV según las variables: autores, título de documento, año, título de la fuente/revista, autores con filiaciones, resumen y tipo de documento.

En SciELO Perú, se ubicaron las revistas de la categoría «Ciencias de la Salud». En la fecha de búsqueda, esta base incluía doce revistas. Para recuperar estas publicaciones, se revisaron todos los números de las revistas publicados en el portal de Scielo Perú. Se revisó manualmente la versión digitalizada de cada publicación y se recuperaron aquellas con al menos un autor con filiación del INS. Dicha identificación fue realizada por dos analistas con experiencia en estudios bibliométricos (DF y MC).

\section{Unidad de análisis}

La unidad de análisis fue la publicación original. Para los registros de Scielo Perú se consideraron los siguientes tipos de documentos: artículo original, artículo original breve y comunicación corta. Para Scopus se revisó manualmente los registros para validarlo como publicaciones que reportaran resultados de un estudio o proyecto de investigación, siguiendo o no la estructura: introducción, métodos, resultados y discusión.

\section{Elaboración de base de datos}

En Scopus se recuperaron 664 publicaciones, se eliminaron tres por repetición y doce por no contar con algún autor con filiación del INS. Tras la depuración quedaron 649 registros. A partir de la recuperación manual de Scielo Perú se recopilaron datos de 621 publicaciones.

En Scopus se encontraron indizados: 508 artículos («Article»), 57 cartas al editor («Letter»), 34 editoriales («Editorial»), 33 revisiones («Review»), 10 notas, 4 «Conference Paper», 2 erratas y 1 artículo en prensa. De Scielo Perú, 266 fueron identificados como artículos originales, 65 como cartas al editor, 
50 como editoriales, 45 como artículos de revisión, 39 como comunicaciones cortas, 38 como artículos originales breves, 37 como artículos especiales, 28 como artículos de conferencia, 19 como galerías fotográficas, 16 como reportes de caso, 14 como artículos históricos/biográficos, 2 como notas, 1 como tema de actualidad y 1 documento guía.

Se consolidaron las bases recuperadas de Scopus y de Scielo Perú. Entre 1998 y 2018, las publicaciones de la Revista de Gastroenterología del Perú, de la Revista de Investigaciones Veterinarias del Perú y de la Revista Peruana de Biología, así como las publicaciones entre 2009-2018 de la Revista Peruana de Medicina Experimental y Salud Pública (RPMESP), generaron registros duplicados. Se compararon los títulos en español y se eliminaron 302 duplicados. La base de datos consolidada comprendió 968 registros.

Se creó una hoja de cálculo consolidada para las publicaciones originales. Debido a que la información exportada de Scopus mostraba errores en la clasificación del documento, se procedió a corroborar manualmente cada registro, revisando la estructura e información del resumen o el artículo completo para validarlo como publicación original. Este proceso fue realizado por dos colaboradores con experiencia en estudios bibliométricos, en caso de discordancia, la definición fue realizada por el autor.

La base de datos de publicaciones originales incluyó las siguientes variables: base de datos de recuperación, nombre de revista científica, año de publicación, título de publicación, resumen, autores, firma institucional, firma país.

Se normalizó manualmente los nombres de los autores debido a que existían datos incompletos, con problemas ortográficos o con diferente denominación para un mismo autor. Para la normalización de la filiación institucional se consideró la institución mayor que comprendiera laboratorio, dirección, instituto, facultad o subunidad.

Para el análisis de citas, en una hoja de cálculo se recopiló el número de citas por año para cada registro con al menos una citación. Se realizó el conteo de citas solo para las publicaciones recuperadas de Scopus a través de su herramienta Metrics. No se consideraron las publicaciones recuperadas de Scielo Perú debido a que su herramienta de conteo de citas aún está en fase de desarrollo y se validó que en muchos casos no arroja datos verídicos.

\section{Variables}

Los indicadores de producción fueron el número de publicaciones originales por año de publicación, revista científica y temática. Los años de publicaciones fueron recategorizados en dos subperiodos (1998-2008 y 2009-2018). La temática fue asignada manualmente por el autor, revisando el título y resumen de cada publicación y tomando como referencias las Prioridades Nacionales de Investigación en Salud en Perú 2019-2023 ${ }^{(11)}$. El índice de productividad fue definido como el logaritmo del número de publicaciones originales por año.
El índice de productividad de autores con filiación del INS fue categorizado en pequeños productores (una sola publicación), medianos (entre dos y nueve publicaciones) y grandes productores ( $\geq 10$ publicaciones) ${ }^{(4)}$.

Los indicadores de colaboración fueron índice de coautoría (número de autores/número de publicaciones originales), índice de número de firmas institucionales por documento (número de firmas institucionales/número de publicaciones originales), tasa de colaboración nacional (publicaciones originales con al menos una firma institucional del Perú que no sea el INS/total de publicaciones originales $\mathrm{x} 100 \%$ ) y tasa de colaboración internacional (porcentaje de publicaciones originales en cuyas firmas institucionales existe al menos una institución extranjera).

El número autores fue recategorizado en único, de dos a cinco, de seis a diez y más de diez; el número de firmas institucionales fue recategorizado en solo INS, dos a tres, cuatro a cinco y seis o más. A partir de la identificación de la revista se consideró nacional (editada en el Perú) o extranjera. La ocurrencia de un primer autor con filiación INS fue usada como un indicador de capacidad de construcción e iniciativa ${ }^{(12)}$, debido a que es aquella posición con mayor contribución intelectual y operativa dentro del estudio ${ }^{(13,14)}$.

A partir del número de citaciones por año se construyeron los siguientes indicadores: citas obtenidas por publicación original, índice citas/publicación original, número de publicaciones originales citados al menos una vez y proporción de documentos citados ${ }^{(4)}$. La velocidad de citación fue definida como el tiempo (en años) transcurridos desde la publicación hasta la primera citación ${ }^{(10)}$.

\section{Análisis de datos}

Los indicadores bibliométricos serán presentados para todo el periodo de estudio y para los subperiodos 1998-2008 y 2009-2018. Los indicadores bibliométricos de producción, colaboración e impacto serán presentados en frecuencias, porcentajes y medidas de tendencia central. Para evaluar las características asociadas a las publicaciones originales con primera autoría del INS se comparó las proporciones mediante chi cuadrado y se calculó la razón de proporciones con intervalo de confianza al 95\%. Se calculó el índice $\mathrm{H}$ para la producción del INS, y el análisis estadístico se realizó en Microsoft Excel y Epidat 3.1.

\section{RESULTADOS}

\section{Indicadores de producción}

En el periodo de estudio, 618 publicaciones originales con al menos un autor con filiación del INS fueron recuperadas de Scopus y Scielo Perú. Se observó un crecimiento de la producción científica de tipo polinómico de segundo grado (Figura 1). El 55,9\% (346/618) de las publicaciones fue en re- 


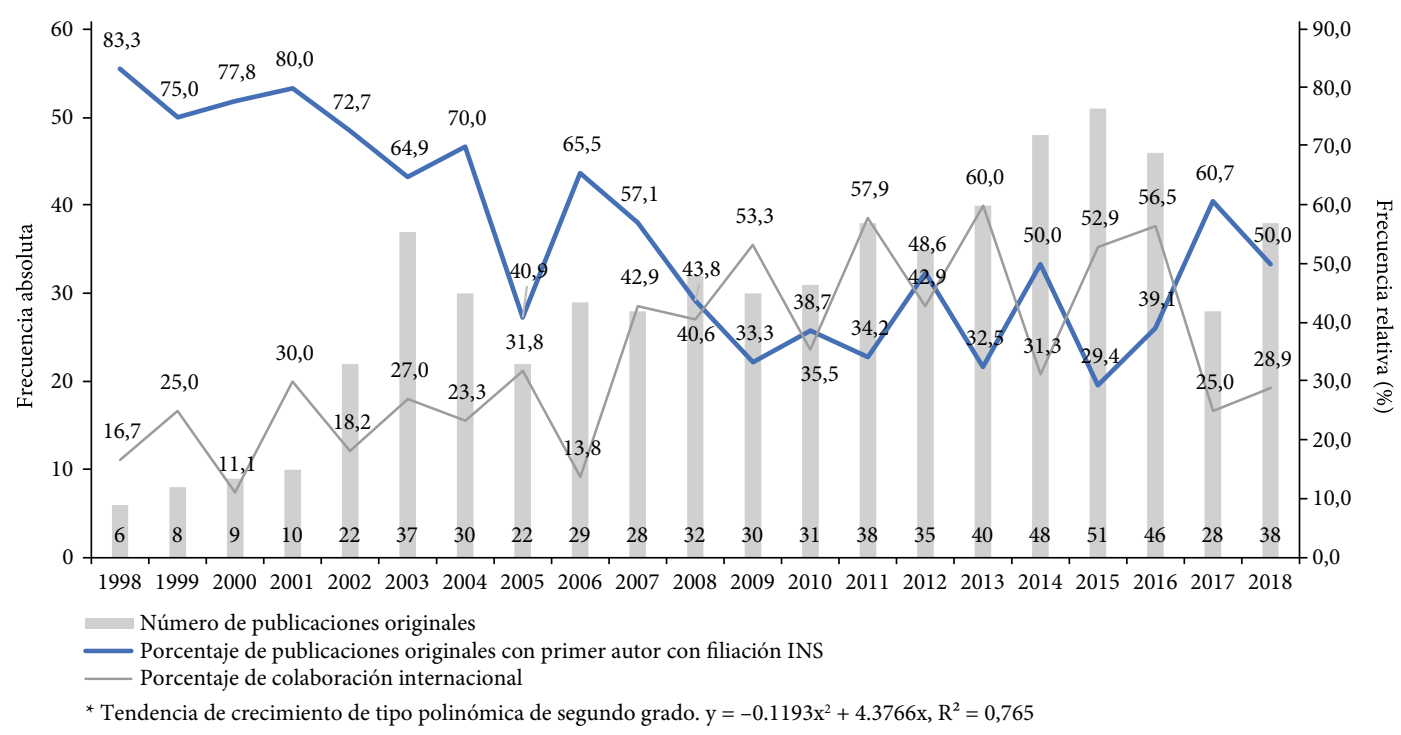

Figura 1. Evolución de publicaciones originales *, primera autoría del Instituto Nacional de Salud y de colaboración internacional, periodo 1998-2018

vistas peruanas. Entre 1998 y 2008, el 26,6\% (62/233) de las publicaciones fue en revistas internacionales, mientras que entre el 2009 y el 2018, este porcentaje fue 54,5\% (210/385). Para todo el periodo, 136 revistas diferentes publicaron los artículos. El 77,8\% de las publicaciones estuvo concentrado en 26 revistas (Tabla 1 ).

El índice de productividad tuvo una media (desviación estándar [DE]) para el periodo 1998-2008 de 1,25 (0,28) y entre el 2009 y el 2018 de 1,78 (0,09). Identificamos 440 autores diferentes con filiación del INS; según el índice de productividad, 231 (52,5\%) fueron pequeños productores; 179 $(40,7 \%)$, medianos; y $30(6,8 \%)$, grandes. Al momento de la recuperación de los datos, siete grandes productores ya no contaban con filiación institucional del INS (Figura 2).

\section{Primera autoría}

El 49,0\% (303/618) de las publicaciones tuvo un primer autor con filiación del INS. Entre los dos subperiodos, la primera autoría para el INS se redujo del 62,2\% al 41,0\% (Figura 1). La presencia de un primer autor con filiación del INS es dos veces más frecuente entre las publicaciones sin colaboración internacional (RP: 2,93; IC95\%: 2,29 a 3,74). A medida que se incrementan el número de autores y firmas institucionales la probabilidad que el primer autor tenga filiación INS es menor. La primera autoría con filiación INS se asocia a publicar en revistas nacionales, especialmente en la RPMESP (RP: 2,33; IC95\%: 1,95 a 2,79) (Anexo 1).

\section{Análisis temático}

Ciento treinta y dos $(21,4 \%)$ publicaciones fueron sobre enfermedades transmitidas por vectores, 60 (9,7\%) sobre tu- berculosis y 59 (9,5\%) sobre zoonosis. En 24 de los 29 temas, la producción se incrementó en el segundo subperiodo: la tuberculosis pasó de 16 a 44; la alimentación y nutrición, de 12 a 30; las enfermedades no transmisibles, de uno a 24 ; los antivenenos, de cuatro a once; la bibliometría, de uno a 13; entre otros. Entre 1998 y 2008, las enfermedades transmitidas por vectores, tuberculosis y zoonosis, concentraban el $51,5 \%$ de publicaciones, entre el 2009 y el 2018 estos temas concentraron solo el 34,0\% (Tabla 2).

\section{Indicadores de colaboración}

Identificamos 4099 firmas de autor. Para todo el periodo de estudio el indice de coautoria fue 6,63. Además, el 80,3\% de las publicaciones tuvo colaboracion con autores de otra institución. Entre los dos subperiodos, la tasa de colaboración internacional pasó de $27,9 \%$ a 45,5\%, mientras que la colaboración nacional pasó de 65,2\% a 71,2\% (Tabla 3).

En todo el periodo, el INS colaboró con 491 firmas institucionales diferentes provenientes de 42 países (incluido Perú). Entre las firmas institucionales, 182 fueron entidades peruanas; 68, de los Estados Unidos; 30, de Brasil; 25, de Argentina; y 18, de Ecuador. En dichas interacciones institucionales, se dieron 2679 colaboraciones con autores con filiación de alguna institución peruana; 806 con autores con filiación de instituciones de los Estados Unidos; 395, con instituciones de Brasil; 113, con instituciones de Argentina; y 81 con instituciones de Ecuador (Anexo 2). Las tres instituciones de mayor interacción con el INS fueron la Universidad Nacional Mayor de San Marcos (UNMSM), con 319 coautorías, seguido de la Universidad Peruana Cayetano Heredia 
Tabla 1. Principales revistas científicas en la difusión de publicaciones originales con filiación institucional del Instituto Nacional de Salud, periodo 1998-2018

\begin{tabular}{|c|c|c|c|c|c|c|c|}
\hline \multirow[b]{2}{*}{$\mathbf{N}^{\circ}$} & \multirow[b]{2}{*}{ Revista } & \multirow[b]{2}{*}{ País ${ }^{a}$} & \multicolumn{2}{|c|}{ Subperiodo } & \multicolumn{3}{|c|}{ Total } \\
\hline & & & $\begin{array}{r}1998- \\
2008 \\
\end{array}$ & $\begin{array}{c}2009- \\
2018\end{array}$ & $\mathbf{n}$ & $\%$ & $\%$ acumulado \\
\hline 1 & Revista Peruana de Medicina Experimental y Salud Pública & Perú & 158 & 125 & 283 & 45,8 & 45,8 \\
\hline 2 & Anales de la Facultad de Medicina & Perú & 9 & 29 & 38 & 6,1 & 51,9 \\
\hline 3 & American Journal of Tropical Medicine and Hygiene & EE. UU. & 12 & 7 & 19 & 3,1 & 55,0 \\
\hline 4 & PLoS ONE & EE. UU. & 1 & 12 & 13 & 2,1 & 57,1 \\
\hline 5 & Emerging Infectious Diseases & EE. UU. & 3 & 9 & 12 & 1,9 & 59,1 \\
\hline 6 & International Journal of Tuberculosis and Lung Disease & Francia & 6 & 5 & 11 & 1,8 & 60,8 \\
\hline 7 & Acta Tropica & Holanda & 0 & 9 & 9 & 1,5 & 62,3 \\
\hline 8 & PLoS Neglected Tropical Diseases & EE. UU. & 0 & 8 & 8 & 1,3 & 63,6 \\
\hline 9 & Genome Announcements & EE. UU. & 0 & 7 & 7 & 1,1 & 64,7 \\
\hline 10 & Revista de Salud Pública & Colombia & 1 & 6 & 7 & 1,1 & 65,9 \\
\hline 11 & Toxicon & Reino Unido & 0 & 7 & 7 & 1,1 & 67,0 \\
\hline 12 & Malaria Journal & Reino Unido & 0 & 6 & 6 & 1,0 & 68,0 \\
\hline 13 & Revista de Gastroenterología del Perú & Perú & 2 & 4 & 6 & 1,0 & 68,9 \\
\hline 14 & Revista Médica Herediana & Perú & 0 & 6 & 6 & 1,0 & 69,9 \\
\hline 15 & Memorias do Instituto Oswaldo Cruz & Brasil & 3 & 2 & 5 & 0,8 & 70,7 \\
\hline 16 & BMC Infectious Diseases & Reino Unido & 0 & 4 & 4 & 0,6 & 71,4 \\
\hline 17 & Clinical Infectious Diseases & Reino Unido & 3 & 1 & 4 & 0,6 & 72,0 \\
\hline 18 & Horizonte Médico (Lima) & Perú & 0 & 4 & 4 & 0,6 & 72,7 \\
\hline 19 & International Journal of Infectious Diseases & Holanda & 0 & 4 & 4 & 0,6 & 73,3 \\
\hline 20 & Journal of Medical Entomology & EE. UU. & 0 & 4 & 4 & 0,6 & 73,9 \\
\hline 21 & Nutrición Clinica y Dietetica Hospitalaria & España & 0 & 4 & 4 & 0,6 & 74,6 \\
\hline 22 & Revista da Sociedade Brasileira de Medicina Tropical & Brasil & 2 & 2 & 4 & 0,6 & 75,2 \\
\hline 23 & Revista de Investigaciones Veterinarias del Perú & Perú & 0 & 4 & 4 & 0,6 & 75,9 \\
\hline 24 & Revista do Instituto de Medicina Tropical de Sao Paulo & Brasil & 1 & 3 & 4 & 0,6 & 76,5 \\
\hline 25 & Revista Panamericana de Salud Pública & EE. UU. & 1 & 3 & 4 & 0,6 & 77,2 \\
\hline 26 & Revista Peruana de Biología & Perú & 2 & 2 & 4 & 0,6 & 77,8 \\
\hline
\end{tabular}

a País de casa editora de revista según Scimago Journal \& Country Rank

(UPCH), con 307, y el Centro de Prevención y Control de Enfermedades de los Estados Unidos, con 274 (Anexo 3).

Figura 2. Distribución de la producción de publicaciones originales entre autores con filiación del Instituto Nacional de Salud, periodo 19982018

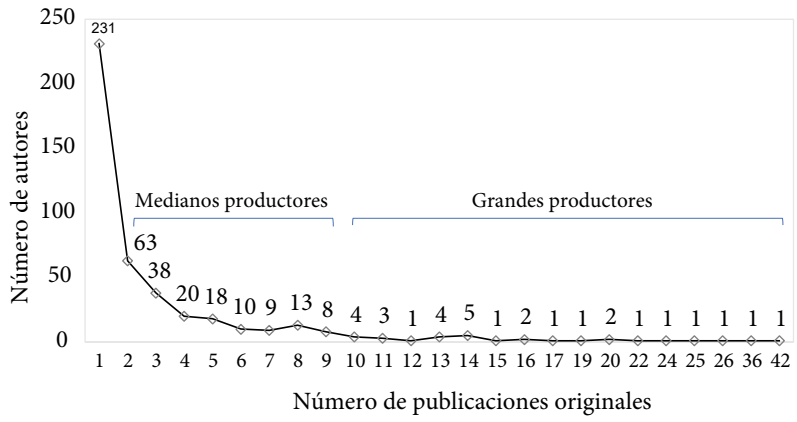

\section{Análisis de citaciones}

De 407 publicaciones originales recuperadas de Scopus, 339 $(83,3 \%)$ fueron citadas al menos una vez. El número de citaciones fue de 5206. Las 65 publicaciones entre 1998 y 2008 generaron 325 citas, mientras que las 342 publicaciones del periodo 2009-2018 generaron 4881. El índice citas/documentos para todo el periodo fue 12,8 (Tabla 4). Entre el 2008 y el 2015, los artículos publicados en cada año de dicho periodo recibieron 400 o más citaciones desde su publicación (Anexo 4).

Entre las publicaciones con alguna citación, 106 (31,3\%) fueron citadas en el mismo año de publicación; 151 (44,5\%), luego de un año; 46 (13,6\%), luego de dos años; 17 (5,0\%), a los tres; 7 (2,1\%), a los cuatro años; $5(1,5 \%)$, a los cinco años; tres $(0,9 \%)$, a los seis años; dos $(0,6 \%)$, a los siete años; una $(0,3 \%)$, a los ocho años; y una $(0,3 \%)$, a los 10 años. 
Tabla 2. Temática de la producción científica de publicaciones originales con participación del Instituto Nacional de Salud, periodo 1998-2018

\begin{tabular}{|c|c|c|c|c|c|c|}
\hline \multirow{2}{*}{ Tema } & \multicolumn{2}{|c|}{ 1998-2008 } & \multicolumn{2}{|c|}{ 2009-2018 } & \multicolumn{2}{|c|}{ Total } \\
\hline & $\mathbf{n}$ & $\%^{a}$ & $\mathbf{n}$ & $\%^{a}$ & $\mathbf{n}$ & $\%$ \\
\hline Enfermedades transmitidas por vectores & 78 & 33,5 & 54 & 14,0 & $132^{\mathrm{b}}$ & 21,4 \\
\hline Tuberculosis & 16 & 6,9 & 44 & 11,4 & 60 & 9,7 \\
\hline Zoonosis & 26 & 11,2 & 33 & 8,6 & $59^{c}$ & 9,5 \\
\hline Alimentación y nutrición & 12 & 5,2 & 30 & 7,8 & 42 & 6,8 \\
\hline Otras enfermedades transmisibles infecciosas & 20 & 8,6 & 13 & 3,4 & 33 & 5,3 \\
\hline Microbiología & 16 & 6,9 & 16 & 4,2 & 32 & 5,2 \\
\hline Enfermedades no transmisibles & 1 & 0,4 & 23 & 6,0 & 24 & 3,9 \\
\hline Entomología & 6 & 2,6 & 15 & 3,9 & 21 & 3,4 \\
\hline Infecciones por retrovirus & 10 & 4,3 & 8 & 2,1 & 18 & 2,9 \\
\hline Recursos humanos en salud & 3 & 1,3 & 13 & 3,4 & 16 & 2,6 \\
\hline Antivenenos & 4 & 1,7 & 11 & 2,9 & 15 & 2,4 \\
\hline Salud del niño y adolescente & 3 & 1,3 & 12 & 3,1 & 15 & 2,4 \\
\hline Bibliometría & 1 & 0,4 & 13 & 3,4 & 14 & 2,3 \\
\hline Enfermedades nutricionales y metabólicas & 2 & 0,9 & 12 & 3,1 & 14 & 2,3 \\
\hline Otros $^{d}$ & 2 & 0,9 & 12 & 3,1 & 14 & 2,3 \\
\hline Hepatitis viral & 5 & 2,1 & 7 & 1,8 & 12 & 1,9 \\
\hline Micosis & 10 & 4,3 & 2 & 0,5 & 12 & 1,9 \\
\hline Servicios de salud & 2 & 0,9 & 10 & 2,6 & 12 & 1,9 \\
\hline Salud mental & 1 & 0,4 & 9 & 2,3 & 10 & 1,6 \\
\hline Productos farmacéuticos & 3 & 1,3 & 6 & 1,6 & 9 & 1,5 \\
\hline Salud ocupacional & 2 & 0,9 & 7 & 1,8 & 9 & 1,5 \\
\hline Violencia & 1 & 0,4 & 7 & 1,8 & 8 & 1,3 \\
\hline Infecciones respiratorias virales & 2 & 0,9 & 5 & 1,3 & 7 & 1,1 \\
\hline Plantas medicinales & 2 & 0,9 & 4 & 1,0 & 6 & 1,0 \\
\hline Salud sexual y reproductiva & 0 & 0,0 & 6 & 1,6 & 6 & 1,0 \\
\hline Gestión y ética de investigación & 1 & 0,4 & 4 & 1,0 & 5 & 0,8 \\
\hline Metales pesados & 0 & 0,0 & 5 & 1,3 & 5 & 0,8 \\
\hline Sistemas de información en salud & 2 & 0,9 & 3 & 0,8 & 5 & 0,8 \\
\hline Salud en comunidades indígenas & 2 & 0,9 & 1 & 0,3 & 3 & 0,5 \\
\hline
\end{tabular}

a Proporción respecto a número de publicaciones originales del periodo correspondiente. ${ }^{\mathrm{b}}$ Incluyen subtemas: malaria (38), leishmaniasis (26), dengue (24), enfermedad de Chagas (18), bartonelosis humana (10), fiebre amarilla (8), otros arbovirus (4) y síndrome febril agudo (4). ${ }^{\mathrm{c}}$ Incluyen subtemas: leptospirosis (14), rickettsiosis (10), equinococosis (8), rabia (7), hidatidosis (5), peste (4), fascioliasis (3), brucelosis (2), toxocariosis (2), cisticercosis (2), infecciones por Borrelia (1) y ehrlichiosis (1). ${ }^{\mathrm{d}}$ Incluye reflujo gastroesofágico, medicina alternativa, cambio climático, zoología, vacunación, accidentes de tránsito, guías de práctica clínica, genómica, embolismo pulmonar, bioinformática, microbioma

Las publicaciones originales con filiación del INS tuvieron un índice $\mathrm{H}$ de 37 . El artículo con más citaciones fue publicado el 2011 y alcanzó 176 citas. En dicho artículo el autor de filiación INS ocupó el lugar 12 de autoría. Cuatro publicaciones recibieron más de 100 citaciones, en ninguno de ellos, el primer autor tuvo filiación del INS. De las $35 \mathrm{pu}$ blicaciones más citadas ( $\geq 40$ citaciones), tres $(8,6 \%)$ tuvieron al primer autor con filiación INS (Anexo 5).

\section{DISCUSIÓN}

La producción de publicaciones originales con participación del INS ha tenido una tendencia creciente de 1998 hasta
2015, y hubo un crecimiento del $65,2 \%$ en el número de publicaciones en el decenio 2009-2018. El INS ha contribuido con 618 publicaciones originales, y si consideramos todos los tipos de publicaciones la contribución, llegaría a casi mil artículos. Al tomar como denominador las 6032 publicaciones biomédicas con participación de alguna institución peruana encontradas entre 1997 y 2016 en la base WoS (2), entre el $10 \%$ y el $15 \%$ de la producción científica biomédica del Perú tendría participación del INS. Para interpretar dicha estimación, debemos considerar que analizamos una base de datos diferente y dos años adicionales.

De 2016 al 2018 se redujo el número de publicaciones, existirían varias explicaciones plausibles: a) la menor gene- 
Tabla 3. Indicadores bibliométricos de colaboración del Instituto Nacional de Salud, periodo 1998-2018

\begin{tabular}{|c|c|c|c|}
\hline \multirow{2}{*}{ Indicador } & \multicolumn{2}{|c|}{ Periodo } & \multirow{2}{*}{ Total } \\
\hline & 1998-2008 & $2009-2018$ & \\
\hline Número de publicaciones originales (a) & 233 & 385 & 618 \\
\hline Número de firmas de autor (b) & 1370 & 2729 & 4099 \\
\hline Número de firmas institucionales (c) & 618 & 1426 & 2044 \\
\hline Índice de coautoría (b/a en \%) & 5,9 & 7,1 & 6,6 \\
\hline Índice de número de firmas institucionales (c/a en \%) & 2,7 & 3,7 & 3,3 \\
\hline Número de PO con al menos una institución con firma país extranjera (d) & 65 & 175 & 240 \\
\hline Tasa de colaboración internacional (d/a en \%) & 27,9 & 45,5 & 38,8 \\
\hline Número de PO con al menos una institución (diferente al INS) con firma país Perú (e) & 152 & 274 & 426 \\
\hline Tasa de colaboración nacional (e/a en \%) & 65,2 & 71,2 & 68,9 \\
\hline Número de PO con filiación del INS (sin colaboración) (f) & 58 & 64 & 122 \\
\hline Proporción de PO sin colaboración institucional (f/a en \%) & 24,9 & 16,6 & 19,7 \\
\hline
\end{tabular}

PO: Publicación original

ración de nuevos proyectos de investigación; en el portal web del INS, se muestra que de 44 proyectos financiados para el 2018, solo uno fue aprobado en dicho año y diez en el 2017; de los 31 proyectos financiados para el 2019, cinco fueron aprobados ese mismo año, los demás fueron genera- dos y aprobados en años previos; b) la emigración de grandes productores, al 2019 casi la cuarta parte de estos autores ya no tenían la filiación del INS; y c) la no culminación de proyectos de investigación, por problemas administrativos y logísticos en la compra de bienes y servicios para las acti-

Tabla 4. Análisis de citaciones de publicaciones originales del Instituto Nacional de Salud del Perú, según Scopus a para el periodo 1999-2018

\begin{tabular}{|c|c|c|c|c|c|}
\hline Año & $\begin{array}{l}\text { Número de PO } \\
\text { (a) }\end{array}$ & $\begin{array}{c}\text { Número de PO citados al menos } \\
\text { una vez } \\
\text { (b) }\end{array}$ & $\begin{array}{c}\text { Proporción de PO } \\
\text { citados } \\
\text { (b/a en \%) }\end{array}$ & $\begin{array}{c}\text { Número de citaciones recibi- } \\
\text { das por todas las PO en cada } \\
\text { año (c) }\end{array}$ & $\begin{array}{l}\text { Índice de citas/ } \\
\text { publicación } \\
\text { (c/a) }\end{array}$ \\
\hline 1999 & 1 & 1 & 100,0 & 0 & 0,0 \\
\hline 2000 & 2 & 1 & 50,0 & 1 & 0,5 \\
\hline 2001 & 2 & 1 & 50,0 & 3 & 1,5 \\
\hline 2002 & 6 & 5 & 83,3 & 1 & 0,2 \\
\hline 2003 & 9 & 9 & 100,0 & 8 & 0,9 \\
\hline 2004 & 7 & 7 & 100,0 & 27 & 3,9 \\
\hline 2005 & 7 & 7 & 100,0 & 41 & 5,9 \\
\hline 2006 & 6 & 5 & 83,3 & 61 & 10,2 \\
\hline 2007 & 11 & 10 & 90,9 & 74 & 6,7 \\
\hline 2008 & 14 & 13 & 92,9 & 109 & 7,8 \\
\hline 2009 & 30 & 25 & 83,3 & 134 & 4,5 \\
\hline 2010 & 30 & 30 & 100,0 & 196 & 6,5 \\
\hline 2011 & 33 & 30 & 90,9 & 301 & 9,1 \\
\hline 2012 & 30 & 27 & 90,0 & 359 & 11,9 \\
\hline 2013 & 37 & 35 & 94,6 & 405 & 10,9 \\
\hline 2014 & 43 & 40 & 93,0 & 527 & 12,3 \\
\hline 2015 & 46 & 41 & 89,1 & 583 & 12,7 \\
\hline 2016 & 38 & 29 & 76,3 & 743 & 19,6 \\
\hline 2017 & 25 & 16 & 64,0 & 856 & 34,2 \\
\hline 2018 & 30 & 7 & 23,3 & 777 & 25,9 \\
\hline Total & 407 & 339 & 83,3 & 5206 & 12,8 \\
\hline
\end{tabular}

${ }^{a}$ Citas recibidas a octubre del 2019, PO: Publicación original 
vidades de investigación ${ }^{(17)}$. A menor cantidad de estudios generados, conducidos y concluidos, menor cantidad de publicaciones que difundan sus resultados.

Las aproximaciones a la producción científica del INS se realizaron en estudios que midieron la producción nacional. Por ejemplo, entre el 2000 y el 2009, se identificaron 65 publicaciones en la base ISI Web of Knowledge ${ }^{(3)}$, mientras que, entre 1997 y 2016, se encontraron 196 publicaciones con filiación INS en WoS ${ }^{(2)}$. Ambos análisis recuperaron artículos de diversa tipología. En nuestro estudio, considerando solo publicaciones originales, encontramos cuatro y tres veces las cantidades reportadas para dichos periodos, por los estudios mencionados. Esta diferencia podría explicarse por la base de datos usada, WoS tiene criterios selectivos de inclusión de sus revistas. En 2014 el número de revistas indizadas en WoS fue 12 000, mientras que en Scopus fue 21 921, además, 11738 revistas estuvieron indizadas en Scopus pero no en WoS $^{(10)}$.

La productividad de los autores del INS sigue la ley de Lotka. Casi la mitad tiene baja productividad y 30 autores fueron los más productivos, ello significa que un porcentaje significativo de investigadores trabajaron en un proyecto de investigación y no volvieron a involucrase en el proceso investigación-publicación, por ende, no continuaron la línea de investigación iniciada ${ }^{(15)}$. Esta característica no permite consolidar expertos en líneas temáticas, ni fomenta la colaboración en dichas áreas ${ }^{(16)}$. Otra explicación sería la baja cultura de publicación. Entre el 2004 y el 2008, solo 18 de 129 proyectos de investigación del INS culminaron en publicación científica ${ }^{(17)}$. Otro estudio mostró que 79 (26,7\%) de 296 resúmenes enviados a un congreso internacional de enfermedades tropicales, culminaron con una publicación científica en un periodo de seis años ${ }^{(18)}$.

En investigación biomédica la autoría suele ser asignada en orden decreciente a la contribución: el primer autor es aquel con mayor aporte en las actividades científicas del estudio y ejerce el liderazgo del proyecto ${ }^{(12,19)}$. Se encontró que la primera autoría del INS se redujo en el último decenio, lo cual estaría asociado con el incremento de los índices de colaboración internacional y un rol secundario de los autores del INS. En estudios colaborativos, los autores de países de ingresos medios o bajos suelen ser relegados en el orden de autoría, debido a que su contribución se limita al reclutamiento de sujetos de estudio, a la recolección de datos, remisión de muestras y otros aspectos técnicos ${ }^{(20,21)}$.

Como ocurre para otras instituciones de investigación ${ }^{(22,23)}$, la red de colaboración internacional del INS se ha expandido en el decenio 2009-2018. Las firmas institucionales provinieron en mayor frecuencia de los Estados Unidos, seguido de Brasil y Argentina. Esta tendencia también ha sido observada en instituciones similares, como el Instituto Fiocruz de Brasil ${ }^{(16)}$. La frecuente colaboración de instituciones peruanas con otras procedentes de los Estados Unidos ha sido reportada en publicaciones médicas entre el 2000 y el 2009; aquellas con más par- ticipación fueron la Universidad Johns Hopkins, el Centro de Control de Enfermedades y la Universidad de Washington ${ }^{(3)}$, el liderazgo de estas instituciones de los Estados Unidos también fue evidente entre 1997 y $2016^{(2)}$.

La colaboración internacional es clave en la investigación científica en Latinoamérica y el campo de la salud pública no es la excepción. Entre 1996 y 2011, las publicaciones científicas en salud pública y de medicina tuvieron un $30 \%$ de colaboración internacional, con una reducción de 35,8\% a $24,5 \%$ entre 1996 y $2011^{(24)}$. En nuestro estudio, la colaboración internacional fue cercana al $40 \%$, con un incremento para el decenio 2009-2018. En instituciones peruanas, la colaboración internacional es variable: en el periodo 20062011, se encontró en el 74,4\% de publicaciones de la UPCH y en el 56,2\% de la UNMSM, proporciones superiores al 90\% se vieron en institutos públicos de investigación ${ }^{(1)}$.

Los artículos con primera autoría del INS son publicados principalmente en la RPMESP. Este escenario se debería a que el INS es la institución editora de esta revista, porque es editada en español y es la única revista peruana de salud pública indizada en Scopus y Medline. Un aspecto negativo de esta preferencia es que la RPMESP no se encuentra indizada en WoS y no publica regularmente en inglés, lo cual limita la posibilidad de citación de sus artículos, de hecho la RPMESP alcanzó solo 945 citaciones en el periodo 2010-2017 ${ }^{(9)}$.

Los temas principales fueron las enfermedades infecciosas, hecho congruente con los dos tercios de artículos al respecto en publicaciones con participación peruana entre 1997-2006 ${ }^{(2)}$. En el rubro de enfermedades infecciosas destaca el crecimiento de la investigación en tuberculosis, cuya producción se triplicó en los subperiodos estudiados. La mayor producción en estos temas se debería a que sus autores provinieron del Centro Nacional de Salud Pública, división del INS dedicada a investigar enfermedades transmisibles y donde se produce la mayor cantidad de proyectos ${ }^{(17)}$.

La producción científica del INS abarcó la mayoría de temas incluidos en las prioridades nacionales de investigación ${ }^{(25)}$ y en las agendas de investigación aprobadas en 2009 y 2011, respectivamente. A pesar de ello, existieron temas desatendidos, por ejemplo, la mortalidad materna no contó con alguna investigación específica, o salud mental que en 20 años tuvo diez publicaciones, nueve de ellas en el último decenio. La reducida producción científica en dichos temas es un problema en el país. Un análisis sobre la producción científica nacional entre el 2011 y el 2014 encontró como temas menos estudiados a mortalidad materna con once artículos originales y salud mental con $28^{(26)}$.

A partir de 2011, las citaciones superan las 300 por año, y el 2017 fue el año donde se realizó la mayor cantidad de citaciones de artículos del INS. Este fenómeno podría explicarse por la indización de la RPMESP en Scopus y por el incremento de la colaboración internacional y la publicación en revistas internacionales ${ }^{(27)}$. Una tercera parte de las publicaciones recibieron alguna cita el mismo año de publicación, y casi la mitad durante el año siguiente, esto refleja una alta 
velocidad de citación; es decir, los resultados de investigación suelen diseminarse rápidamente a la comunidad científica y usados en poco tiempo ${ }^{(10)}$.

Entre las limitaciones, no se realizó el análisis de citaciones de todas las publicaciones originales, solo de aquellas recuperadas de Scopus, lo cual no reflejaría la real usabilidad e impacto de estas publicaciones. Este análisis supone una adecuada atribución de la autoría, lo cual podría afectarse por los cambios en los criterios de autoría ocurridos en las dos décadas de estudio. También está presente la posibilidad que los autores del INS no la hayan reconocido como filiación institucional o sin tener vinculación al INS se la haya reportado como institución ${ }^{(28)}$. No se realizó el análisis de fuentes de financiamiento, debido a la heterogeneidad en los reportes; y el análisis de indicadores que evalúan la diseminación, la producción industrial o el impacto en los servicios o sistemas de salud ${ }^{(29)}$. Por último, este análisis puede no reflejar todas las actividades científicas del INS, pues alrededor del $85 \%$ de proyectos que emprende no se publica ${ }^{(17)}$.

Como fortaleza, este estudio analizó solo publicaciones originales, las cuales son el formato estándar para presentar los resultados de investigación científica, que pueden ser tecnologías, productos, nuevos conceptos o hipótesis. La citación de estos artículos nos aproxima mejor al impacto efectivo de las publicaciones de una institución en el avance de la ciencia ${ }^{(30)}$.

En conclusión, la participación del INS en publicaciones originales en el decenio 2009-2018 se incrementó, a pesar

\section{REFERENCIAS BIBLIOGRÁFICAS}

1. Consejo Nacional de Ciencia, Tecnología e Innovación Tecnológica. Principales indicadores bibliométricos de la actividad científica peruana. 2006-2011 [Internet]. CONCYTEC; 2014 [Citado el 11 de diciembre del 2019]. Disponible en: http://repositorio.concytec. gob.pe/bitstream/20.500.12390/93/1/indicadores_bibliometricos_peru_2006_2011.pdf.

2. Belter CW, Garcia PJ, Livinski AA, Leon-Velarde F, Weymouth KH, Glass RI. The catalytic role of a research university and international partnerships in building research capacity in Peru: A bibliometric analysis. Lee BY, editor. PLOS Negl. Trop. Dis. 2019;13(7):e0007483. doi: https://doi.org/10.1371/journal.pntd.0007483.

3. Huamaní C, Mayta-Tristán P. Producción científica peruana en medicina y redes de colaboración, análisis del Science Citation Index 2000-2009. Rev Peru Med Exp Salud Publica. 2010;27(3):315-25.

4. Jimenez J, Argimon Pallas JM, Martín Zurro A, Vilardell Tarrés M. Capítulo 19: Bibliometría e indicadores de actividad científica. En: Publicación Científica Biomédica Cómo escribir y publicar un artículo de investigación. Segunda edición. Barcelona, España: Elsevier España; 2016.

5. Mayta-Tristán P, Huamaní C, Montenegro-Idrogo Juan JJ, Samanez-Figari C, González-Alcaide G. Producción científica y redes de colaboración en cáncer en el Perú 2000-2011: un estudio bibliométrico en Scopus y Science Citation Index. Rev Peru Med Exp Salud Publica. 2013; 30(1): 31-36.

6. Caballero P, Gutiérrez C, Rosell G, Yagui M, Alarcón J, Espinoza M, et al. Análisis bibliométrico de la producción científica sobre VIH/ de una caída de la producción en los tres últimos años de observación. La contribución ha sido principalmente en enfermedades transmitidas por vectores, tuberculosis y zoonosis. La creciente colaboración internacional ha generado más publicaciones en revistas extranjeras y cada vez más citadas, pero con reducción de la primera autoría para el INS. Se recomienda consolidar la colaboración internacional, pero con mayor liderazgo del INS; incrementar la masa de grandes productores; consolidar las nuevas áreas temáticas en las cuales investigadores del INS se están posicionando; e incrementar su tasa de colaboración a nivel nacional, con igual esmero que la internacional.

Agradecimientos: A Joselyn Quispe, Diego Flores y Maricela del Carmen Castillo por su trabajo en la construcción, limpieza y procesamiento de la base de datos. A Paolo Wong Chero por la revisión crítica del manuscrito.

Contribuciones de los autores: FR conceptualizó y diseñó el estudio, revisó y validó la base de datos, realizó el análisis de la información, redactó el manuscrito, aprobó la versión final y asume la responsabilidad de su contenido.

Financiamiento: El estudio fue financiado por el Instituto Nacional de Salud.

Conflicto de interés: FR se desempeña como director ejecutivo de la Oficina de Transferencia Tecnológica y Capacitación del Instituto Nacional de Salud. Además, es editor científico de la Revista Peruana de Medicina Experimental y Salud Pública.

Material suplementario: Disponible en la versión electrónica de la RPMESP.

SIDA en el Perú 1985 - 2010. Rev Peru Med Exp Salud Publica. 2011;28(3):470-6.

7. Yagui-Moscoso M, Oswaldo-Jave H, Curisinche-Rojas M, Gutiérrez C, Romaní-Romaní F. Análisis bibliométrico de la investigación sobre tuberculosis en el Perú, periodo 1981-2010. An Fac Med. 2013;73(4):299.

8. Romaní F, Huamani C, González-Alcaide G. Estudios bibliométricos como línea de investigación en las ciencias biomédicas: una aproximación para el pregrado. CIMEL. 2011;14(1):52-62.

9. Romaní F, Cabezas C. Indicadores bibliométricos de las publicaciones científicas de la Revista Peruana de Medicina Experimental y Salud Pública, 2010-2017. Rev Peru Med Exp Salud Publica. 2018;35(4):620. doi: 10.17843/rpmesp.2018.354.3817.

10. Todeschini R, Baccinni A. Handbook of bibliometric indicators. Quantitative Tools for Studying and Evaluating Research. Germany: Wiley-VCH; 2016.

11. Ministerio de Salud. Prioridades Nacionales de Investigación en Salud en Perú (2019-2023). Resolución Ministerial № 658-2019/MINSA [Internet]. [Citado el 27 de marzo 2020]. Disponible en: https://cdn. www.gob.pe/uploads/document/file/343478/Resoluci\%C3\%B3n_ Ministerial_N_658-2019-MINSA.PDF.

12. Kelaher M, Ng L, Knight K, Rahadi A. Equity in global health research in the new millennium: trends in first-authorship for randomized controlled trials among low- and middle-income country researchers 1990-2013. Int J Epidemiol. 2016;45(6):2174-2183. doi: 10.1093/ije/ dyw313. 
13. Perneger TV, Poncet A, Carpentier M, et al. Thinker, Soldier, Scribe: cross-sectional study of researchers' roles and author order in the Annals of Internal Medicine. BMJ Open. 2017;7:e013898. doi: 10.1136/ bmjopen-2016-013898.

14. Zbar A, Frank E. Significance of Authorship Position: An Open-Ended International Assessment. Am J Med Sci. Sci. 2011;341(2):106-9. doi: 10.1097/MAJ.0b013e3181f683a1.

15. Fanelli D, Larivière V. Researchers' Individual Publication Rate Has Not Increased in a Century. Dorta-González P, editor. PLoS One. 2016;11(3):e0149504. doi: 10.1371/journal.pone.0149504.

16. Fonseca BPFE, Silva MVPD, Araújo KM, Sampaio RB, Moraes MO. Network analysis for science and technology management: Evidence from tuberculosis research in Fiocruz, Brazil. Lozano S, editor. PLoS One. 2017;12(8):e0181870. doi: 10.1371/journal.pone.0181870.

17. Garro G, Mormontoy H, Yagui M. Gestión y financiamiento de las investigaciones por el Instituto Nacional de Salud, Perú 2004-2008. Rev Peru Med Exp Salud Publica. 2010;27(3):361-6.

18. Beas R, Anduaga-Beramendi A, Rojas-Ortega A, Cisneros-Montoya A, Pereyra-Elías R, Mayta-Tristán P. Publication of Abstracts with Peruvian Affiliation Presented to the Annual Meeting of the American Society of Tropical Medicine and Hygiene 2006-2010. Am J Trop Med Hyg. 2019; 100(4):1029-1032. doi: 10.4269/ajtmh.18-0168.

19. Baerlocher MO, Newton M, Gautam T, Tomlinson G, Detsky AS. The Meaning of Author Order in Medical Research. J Investig Med. 2007;55(4):174-80. doi: 10.2310/6650.2007.06044.

20. Rees CA, Lukolyo H, Keating EM, Dearden KA, Luboga SA, Schutze $\mathrm{GE}$, et al. Authorship in paediatric research conducted in low- and middle-income countries: parity or parasitism? Trop Med Int Health. 2017;22(11):1362-1370. doi: 10.1111/tmi.12966.

21. Smith E, Hunt M, Master Z. Authorship ethics in global health research partnerships between researchers from low or middle income countries and high income countries. BMC Med Ethics. 2014; 15:42. doi: 10.1186/1472-6939-15-42.
22. Parish AJ, Boyack KW, Ioannidis JPA. Dynamics of co-authorship and productivity across different fields of scientific research. Glanzel W, editor. PLoS One. 2018;13(1):e0189742. doi: 10.1371/journal.pone.0189742.

23. Patel VM, Panzarasa P, Ashrafian H, Evans TS, Kirresh A, Sevdalis $\mathrm{N}$, et al. Collaborative patterns, authorship practices and scientific success in biomedical research: a network analysis. J R Soc Med. 2019;112(6):245-257. doi: 10.1177/0141076819851666.

24. Zacca-González G, Chinchilla-Rodríguez Z, Vargas-Quesada B, de MoyaAnegón F. Bibliometric analysis of regional Latin America's scientific output in Public Health through SCImago Journal \& Country Rank. BMC Public Health. 2014; 14:632. doi: 10.1186/1471-2458-14-632.

25. Yagui Martín, Espinoza Manuel, Caballero Patricia, Castilla Teresa, Garro Gladys, Yamaguchi L Patricia et al. Avances y retos en la construcción del sistema nacional de investigación en salud en el Perú. Rev Peru Med Exp Salud Publica. 2010; 27( 3 ): 387-397. Disponible en: http://www.scielo.org. pe/scielo.php?script=sci_arttext\&pid=S1726-46342010000300012\&lng=es.

26. Romani Romani FR, Roque Henríquez J, Vásquez Loarte T, Mormontoy Calvo H, Vásquez Soplopuco H. Análisis bibliométrico de la producción científica sobre las agendas nacionales de investigación en el Perú 2011-2014. An Fac Med. 2016; 77(3):241.

27. Diekhoff T, Schlattmann P, Dewey M. Impact of Article Language in Multi-Language Medical Journals - a Bibliometric Analysis of Self-Citations and Impact Factor. Bornmann L, editor. PLoS One. 2013; 8(10):e76816. doi: 10.1371/journal.pone.0076816.

28. Bachelet VC, Uribe FA, Díaz RA, Vergara AF, Bravo-Córdova F, Carrasco VA, et al. Author misrepresentation of institutional affiliations: protocol for an exploratory case study. BMJ Open. 2019; 9(2):e023983. doi: 10.1136/bmjopen-2018-023983

29. Thonon F, Boulkedid R, Delory T, Rousseau S, Saghatchian M, van Harten W, et al. Measuring the Outcome of Biomedical Research: A Systematic Literature Review. Fanelli D, editor. PLoS One. 2015; 10(4):e0122239. doi: 10.1371/journal.pone.0122239.

30. Aroeira RI,A.R.B. Castanho M. Can citation metrics predict the true impact of scientific papers?. The FEBS journal. 2020. J. doi: 10.1111/febs.15255. 